\title{
Schizophrenia With Obsessive Compulsive Symptoms: A Case Report
}

\author{
Jha $\mathbf{A}^{1}$, Joshi $\mathrm{D}^{2}$ \\ 1. Psychiatrist, Child Guidance Clinic, Kanti Children's Hospital, Kathmandu, Nepal. 2. Associate \\ Professor, Department of Psychiatry, NAMS, Mental Hospital, Lagankhel, Lalitpur, Nepal.
}

E-mail ${ }^{*}$ Corresponding author : urshealer@gmail.com

\begin{abstract}
Obsessive-compulsive disorder/ symptoms may be co-morbid in schizophrenia. The clinical impact of this comorbidity is poor response to anti-psychotic medications. We present a case of $35 \mathrm{yr}$ old female who presented with symptoms suggestive of schizophrenia and later co-morbid obsessive symptom responded well to addition of fluoxetine to antipsychotics. This case study reveals that the identification and treatment of OCD in schizophrenia is very crucial for optimistic outcome.
\end{abstract}

Keywords: Schizophrenia, OC Symptoms, Nepal

\section{INTRODUCTION}

The term "schizo-obsessive disorder", used for the first time in 1994 by Hwang and Opler, and it applies to the diagnosis of schizophrenia with comorbid obsessive-compulsive disorder or obsessive-compulsive symptoms. ${ }^{1}$ Obsessivecompulsive disorder (OCD) is a common comorbidity in schizophrenia, with prevalence rates ranging from $10 \%$ to $30 \% .^{2}$ Patients with schizophrenia and comorbid obsessivecompulsive disorder show a poor attainment of social function and exibits suicidal behaviours more often than patients diagnosed with schizophrenia only. ${ }^{3}$

\section{CASE- HISTORY}

35 year old female patient came with history suggestive of aggressive behaviour predominantly towards others without any provocation associated with self muttering activity and delusion of thought broadcasting from past 1 year. Intermittently assaultive behaviour was seen because she felt that people in the neighborhoods were speaking about her. She was diagnosed as paranoid schizophrenia based on ICD-10 criteria and was admitted and started olanzapine gradually titrated to 20 $\mathrm{mg} /$ day and short course benzodiazepines . With treatment aggression reduced along with hallucinatory behavior, Thus discharged. Following this she started to follow-up on an OPD basis. During her follow up period her delusion of thought broadcasting faded away but gradually she started asking questions repeatedly to family members and seek answers for her questions. such new symptom emergence hindered her functioning despite well controlled psychotic symptoms. On enquiry patients mother said that there is no past history of obsession from childhood. She endorsed having persistent unwanted ideas clinically not amounting to the delusion. Considering it to be obsessive symptom Fluoxetine 20mg/day was added along with her pre-existing antipsychotics. Patient showed a dramatic improvement in nest 4 weeks time after addition of fluoxetine. She attained nearly her premorbid level of functioning. complete blood count, thyroid function test, CT head, were within normal limits.

\section{DISCUSSION:}

In this case, patient was initially diagnosed as Schizophrenia, with antipsychotics there was remission of psychotic symptoms and later patient developed obsessive symptoms hindering her functioning in a broad concept schizo-obsessive disorder was considered. 
However it is often difficult to clinically differentiate obsessions from the delusions of a psychotic process. This is complicated by the Diagnostic and Statistical Manual V (DSM-V) addition of a "lacking insight" specifier of OCD. Recently, there has been renewed interest in the possibility of emergence of obsessive compulsive symptoms following atypical antipsychotic pharmacotherapy, and the subsequent attempts to explain the phenomena. ${ }^{4}$ studies suggest that prefrontal cortex dysfunctions observed in obsessive compulsive disorder (OCD) and schizophrenia are linked. 5 Considering above case, it can be said that Schizo-obsessive disorder though prevalent is not easily recognized. A detailed history is merited in patients especially those not responding adequately to conventional treatment. Emerging neurobiological and genetic evidence suggests that persons with comorbid OCD and schizophrenia may represent a special category of the population. The clinical issues in this case study highlights the importance of identification and treatment of OCD in schizophrenia. Exciting areas for future research include whether or not these "schizoobsessive" are a subtype of schizophrenia, or whether they merely reflect the high comorbidity of the two disorders. 6

\section{CONCLUSION:}

Management of schizophrenia with OC symptoms must include careful consideration of individualized pharmacological approach for optimal outcome.

\section{REFERENCES:}

1. Patel D. D., Laws K. R., Padhi A., Farrow J. M., Mukhopadhaya K., Krishnaiah R., Fineberg N. A. The neuropsychology of the schizo-obsessive subtype of schizophrenia: a new analysis. Psychologi-cal Medicine 2010; 40: 921-933.

2. Fenton WS, McGlashan TH. The prognostic significance of obsessive-compulsive symptoms in schizophrenia. Am J Psychiatry 1986; 143: 437-41.

3. Ongur D., Goff D.C. Obsessive-compulsive symptoms in schizo-phrenia: associated clinical features, cognitive function and med-ication status. Schizophrenia Research 2005; 75: 349-62

4. Tibbo $P$, Kroetsch $M$, Chue P, Warneke $L$. Obsessivecompulsive disorder in schizophrenia. J Psychiatr Res 2000; 34: 139-46.

5. Cavallaro R, Cavedini P, Mistretta P. et al. Basalcortico frontal circuits in schizophrenia and obsessive- compulsive disorder: a controlled, double dissociation study. Biol Psychiatry 2003; 54: 437-43.

6. Maj M, Sartorius N, Okasha A, Zohar J, et al. Obsessivecompulsive disorder, Second Edition,2002 John Wiley E Sons Ltd. ISBN:0-470-84966-5. 